\title{
Kerstin Hitzbleck/Klara Hübner (dir.), Die Grenzen des Netzwerks 1200-1600
}

Ostfildern : Thorbecke, 2014, 272 p., 29,90€.

\section{Gisela Naegle}

\section{OpenEdition}

\section{Journals}

Édition électronique

URL : http://journals.openedition.org/ifha/8399

DOI : 10.4000/ifha.8399

ISSN : 2198-8943

Éditeur

IFRA - Institut franco-allemand (sciences historiques et sociales)

Référence électronique

Gisela Naegle, « Kerstin Hitzbleck/Klara Hübner (dir.), Die Grenzen des Netzwerks 1200-7600 », Revue de I'IFHA [En ligne], Date de recension, mis en ligne le 01 février 2016, consulté le 22 septembre 2020. URL : http://journals.openedition.org/ifha/8399 ; DOI : https://doi.org/10.4000/ifha.8399

Ce document a été généré automatiquement le 22 septembre 2020

(CIFHA 


\section{Kerstin Hitzbleck/Klara Hübner (dir.), Die Grenzen des Netzwerks}

\section{0-1600}

Ostfildern : Thorbecke, 2014, 272 p., 29,90€.

\section{Gisela Naegle}

La publication dirigée par Kerstin Hitzbleck et Klara Hübner est issue d'un colloque consacré aux limites ou "frontières » (Grenzen) du concept scientifique de réseau (Netzwerks). À ses origines, cet outil méthodique fut développé par les sciences sociales, particulièrement par la sociologie et l'anthropologie, par exemple sous forme de la Social Network Analysis (p. 44). Cependant, plusieurs auteurs soulignent à juste titre que les sources médiévales ne se prêtent pas à un transfert direct et à une application telle quelle de ces méthodes. Pour l'époque médiévale, il est impossible de mener des interviews et des enquêtes. Les sources et les données disponibles sont beaucoup plus lacunaires et, souvent, ils ne permettent pas l'emploi des mêmes procédés mathématiques ou statistiques. Néanmoins, la notion de "réseau " et des études affichant cette étiquette ont fait fortune chez les historiens - y compris chez les médiévistes. Selon les directrices du volume, cet état de la question exige des précisions et des réflexions approfondies autour du concept théorique de réseau / Netzwerk. En dépit de définitions et approches parfois divergentes, entre autres, la plupart des auteurs renvoient aux références reconnues et aux études pionnières du genre historiographique de la Netzwerkforschung germanophone dont les travaux de Wolfgang Reinhard, le volume de Gerhard Fouquet et Hans-Jörg Gilomen sur les réseaux dans le commerce européen du Moyen Âge (2010) ou le « Handbuch Netzwerkforschung » (dir. Christian Stegbauer / Roger Häußling, 2010). Plusieurs contributions décrivent le problème que l'interaction entre individus et groupes de personnes est un processus dynamique, que les réseaux sociaux peuvent se modifier très vite et que la reconstruction de leur configuration par les historiens ne saurait présenter qu'un portrait instantané et nécessairement figé. Par définition, celui-ci serait loin de la réalité du passé. 
S'intégrant dans ce questionnement général, le volume contient douze contributions (dont deux se réfèrent au cadre méthodique général) et les conclusions de Christian Hesse. Placés au début, les articles des deux inspiratrices du livre, K. Hitzbleck et K. Hübner, explorent surtout l'arrière-plan théorique. En analysant l'état actuel de la recherche et des notions voisines comme "Verflechtung " (entrelacement) ou, au sens négatif, «Verhedderung » (confusion, enchevêtrement désordonnée), ils formulent des critiques et souhaits. Conformément à l'appel de réfléchir sur les limites de l'applicabilité de l'outillage méthodique de l'étude des réseaux, les autres auteurs présentent des études de cas. En fonction de leurs exemples concrets, les réponses à la question de l'utilité pratique et de la praticabilité méthodique de ce concept sont fort différentes. Vus dans l'ensemble, les textes ont un très bon niveau scientifique. Néanmoins, en présentant des relations bilatérales, des procédés mentaux ou des stratégies politiques, tout en fournissant des études de cas très intéressantes en soi, plusieurs d'eux s'éloignent du sujet central du fonctionnement des réseaux et de leurs frontières. La fin ou la redéfinition de réseaux médiévaux est un sujet très important. Il est examiné pour différents espaces géographiques. Les cours des princes séculiers ou ecclésiastiques étaient un lieu privilégié de la constitution des réseaux et clientèles. Ainsi, Jörg Schwarz présente la perte d'influence du conseiller Johann Waldner (v. 1430-1502) à la cour de l'empereur Frédéric III. Andreas Bihrer décrit le rôle des "partis» à celle des évêques de Constance et leur influence sur les élections épiscopales (1293-1357). Andreas Fischer discute l'exemple des réseaux des cardinaux (XIIIe siècle) et de leurs tendances d'institutionnalisation. L'Italie est au cœur de la contribution de Christoph Dartmann et de l'article de Kristina Odenweller sur l'écriture d'un livre de famille par le juriste et ambassadeur vénitien Giovan Francesco Capodilista (v. 1434). Jessika Nowak donne une analyse très fine et pertinente d'un codex de Roland Talenti, un Milanais qui vivait dans une Normandie redevenue française après avoir été dominée par les Anglais. Elle qualifie ce texte, une collection de quatre discours et 45 lettres, de « dossier de candidature », fortement influencé par les exigences politiques de la guerre de Cent Ans. J. Nowak arrive à la conclusion qu'il s'agit de l'exemple d'un "faux» réseau artificiellement bricolé pour servir les intentions de son auteur, mais qui ne reflète pas ses contacts réels. Ainsi, il s'agirait donc d'un cas où la Netzwerkanalyse au sens traditionnel ne saurait être appliquée. L'article de C. Dartmann traite un sujet qui se prête particulièrement bien à l'analyse de l'action des réseaux: la culture politique des républiques urbaines italiennes et des tentatives de manipulation des élections. Il illustre d'une façon très intéressante le jeu compliqué des groupes d'influence et familles puissantes. Des observations semblables valent pour les contributions de Bastian Walter-Bogedain sur les réseaux de contacts mis en place par des citadins de l'espace suisse et du Oberrhein pendant les guerres de Bourgogne (v.1430-1502) et de Regula Schmid sur le système changeant des alliances et ligues des villes dont particulièrement l'alliance d'Uri, Schwyz et Unterwalden avec le comte de Kyburg (1327) et le Zürcher Bund (1351). Heinrich Speich décrit une courte entreprise militaire des Eidgenossen contre le Sarganserland (1446) et rapproche cette expédition des méthodes de guerre employées au cours des faides. La contribution de Gerald Schwedler est consacrée aux recommandations de Bernard de Clairvaux relatives aux fonctionnements de la mémoire et de l'oubli. Dans ses conclusions, Christian Hesse met en relief plusieurs aspects dont les risques et pièges liés à l'utilisation de collections de lettres, l'existence de réseaux cachés ou potentiels qui pouvaient être activés si la situation l'exigeait, ou le phénomène d'un renoncement 
volontaire à l'intégration dans les réseaux séculiers (par exemple sous forme de l'entrée dans un monastère). Il met également en garde contre les dangers de la reconstruction des Ego-Netzwerke, c'est-à-dire des réseaux centrés sur un individu particulier.

Dans l'ensemble, le volume donne un panorama actuel des débats méthodiques, des limites et des frontières de la Netzwerkforschung germanophone. Ses très riches études de cas sont particulièrement bien fournies pour l'espace helvétique et celui des villes. Vous trouverez la table des matières ici : http://d-nb.info/1054779406/04.

INDEX

Thèmes : Histoire sociale, Historiographie/Méthodologie

Index chronologique : Ouvrages transpériodiques, Moyen Âge

\section{AUTEUR}

GISELA NAEGLE

Université de Gießen/Université Paris 1 